\title{
Synthesis of Hierarchically Porous Silica and Metal Oxide Beads Using Emulsion-Templated Polymer Scaffolds
}

H. Zhang, G. C. Hardy, Y. Z. Khimyak, M. J. Rosseinsky and A. I. Cooper*

Donnan and Robert Robinson Laboratories, Department of Chemistry, University of Liverpool, Crown Street, Liverpool L69 3BX, United Kingdom

* To whom correspondence should be addressed. E-mail: aicooper@liv.ac.uk.

Tel: 0044151 7943548. Fax: 00441517943588

Supporting Information: Figures S1-S10 (11 pages total) 

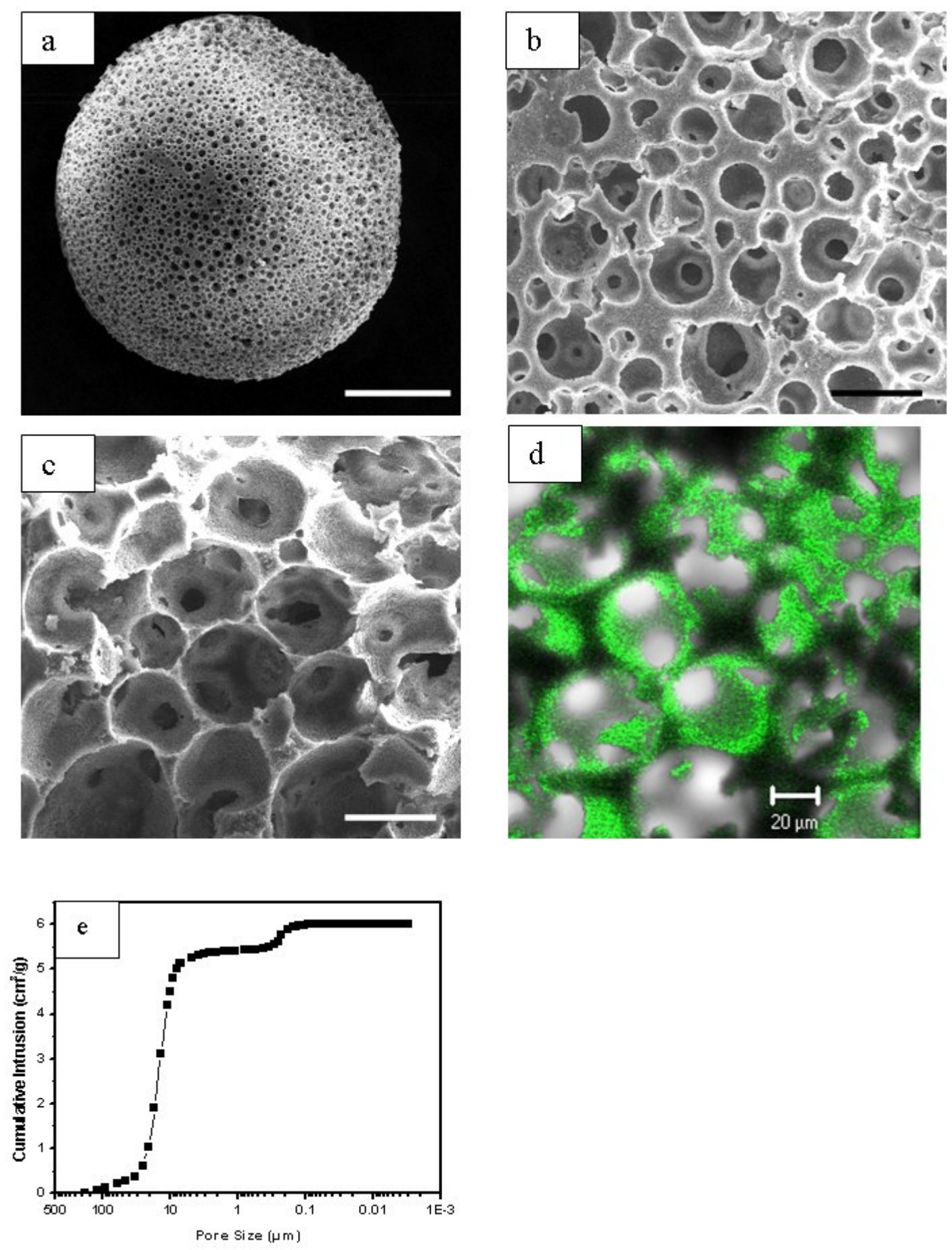

Figure S1: Original PAM Beads (a) Surface of a single bead, scale bar $=500 \mu \mathrm{m}$; (b) Surface shown at higher magnification, scale bar $=50 \mu \mathrm{m}$; (c) Internal structure of one cross-sectioned bead, showing interconnected pores, scale bar $=50 \mu \mathrm{m}$; (d) CLSM image (reflectance mode) corresponding to Fig. 1d in paper text, green colour from the reflectance of polymer; (e) Cumulative intrusion vs pore size, mercury intrusion porosimetry. 

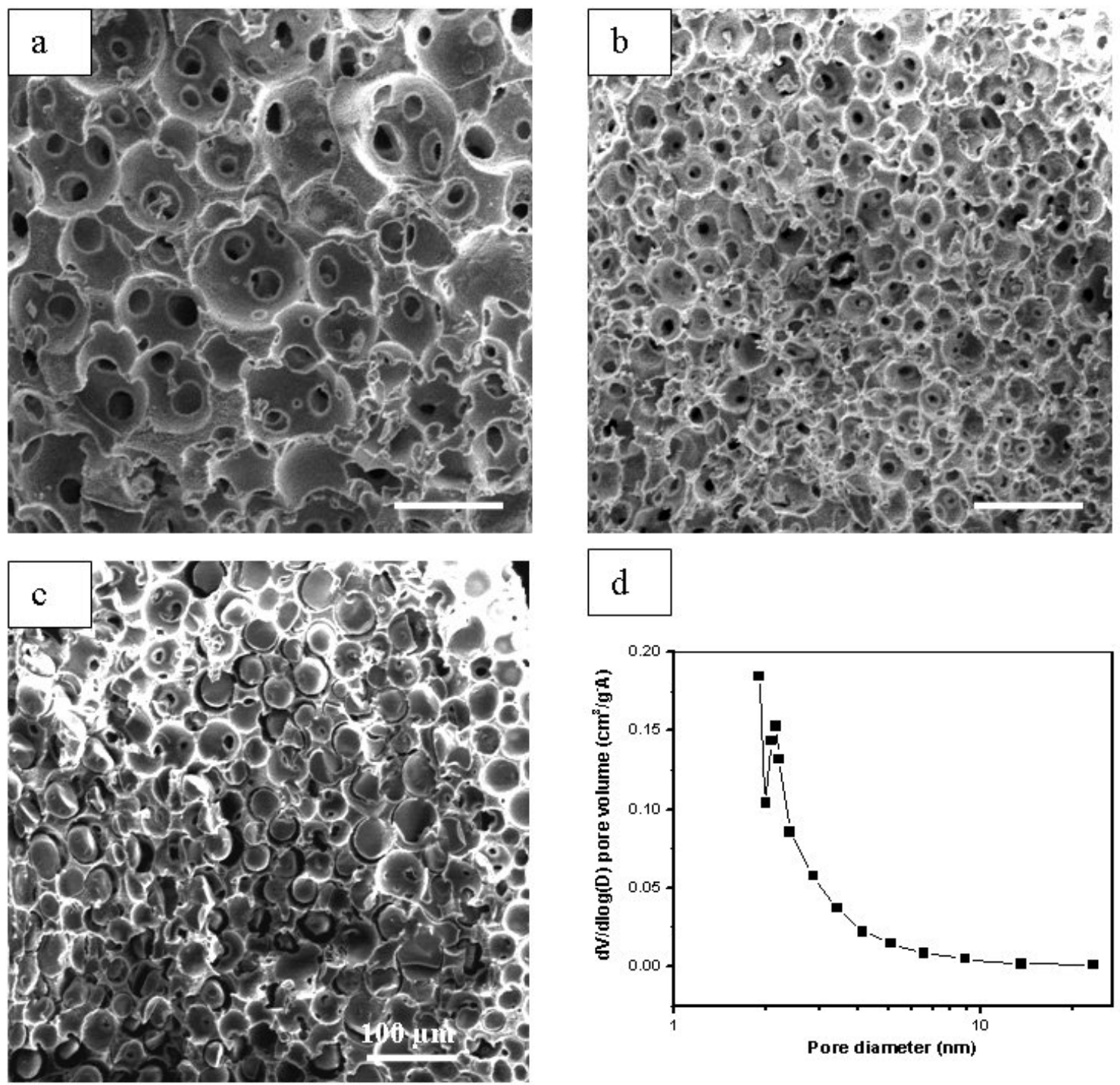

Figure S2: Silica composite beads (a) Internal pore structure of composite bead C-SiO1, scale bar $=50 \mu \mathrm{m}$. (b) Porous surface structure of composite beads C-SiO-2, scale bar $=100 \mu \mathrm{m}$. (c) Internal pore structure of composite beads C-SiO-3. (d) Pore size distribution (C-SiO-4) calculated from $\mathrm{N}_{2}$ sorption data using BJH theory. 

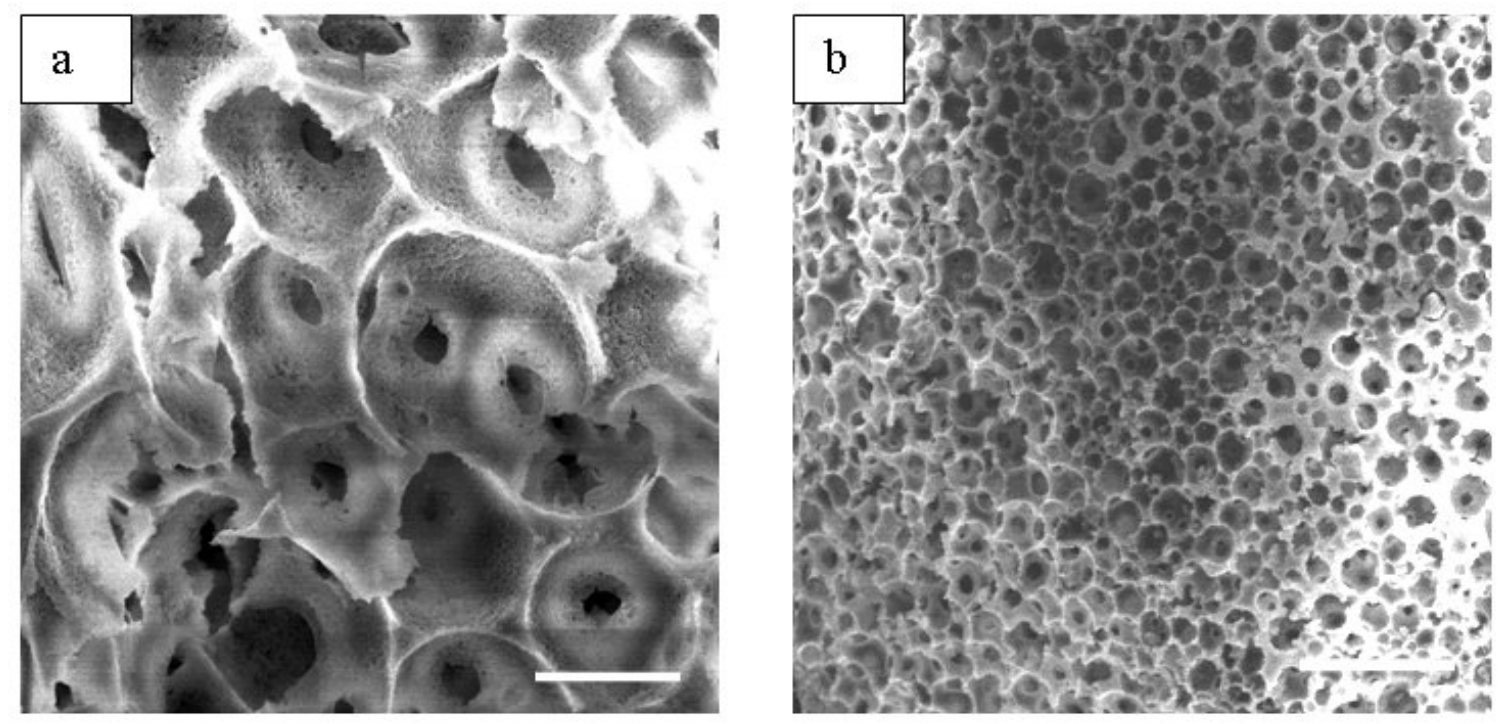

Figure S3: SEM images of calcined silica beads (a) Internal pore structure of silica beads SiO-1, showing the pores are highly interconnected, scale bar $=25 \mu \mathrm{m}$; (b) Highly porous surface structure of silica beads SiO-3, scale bar $=100 \mu \mathrm{m}$. 

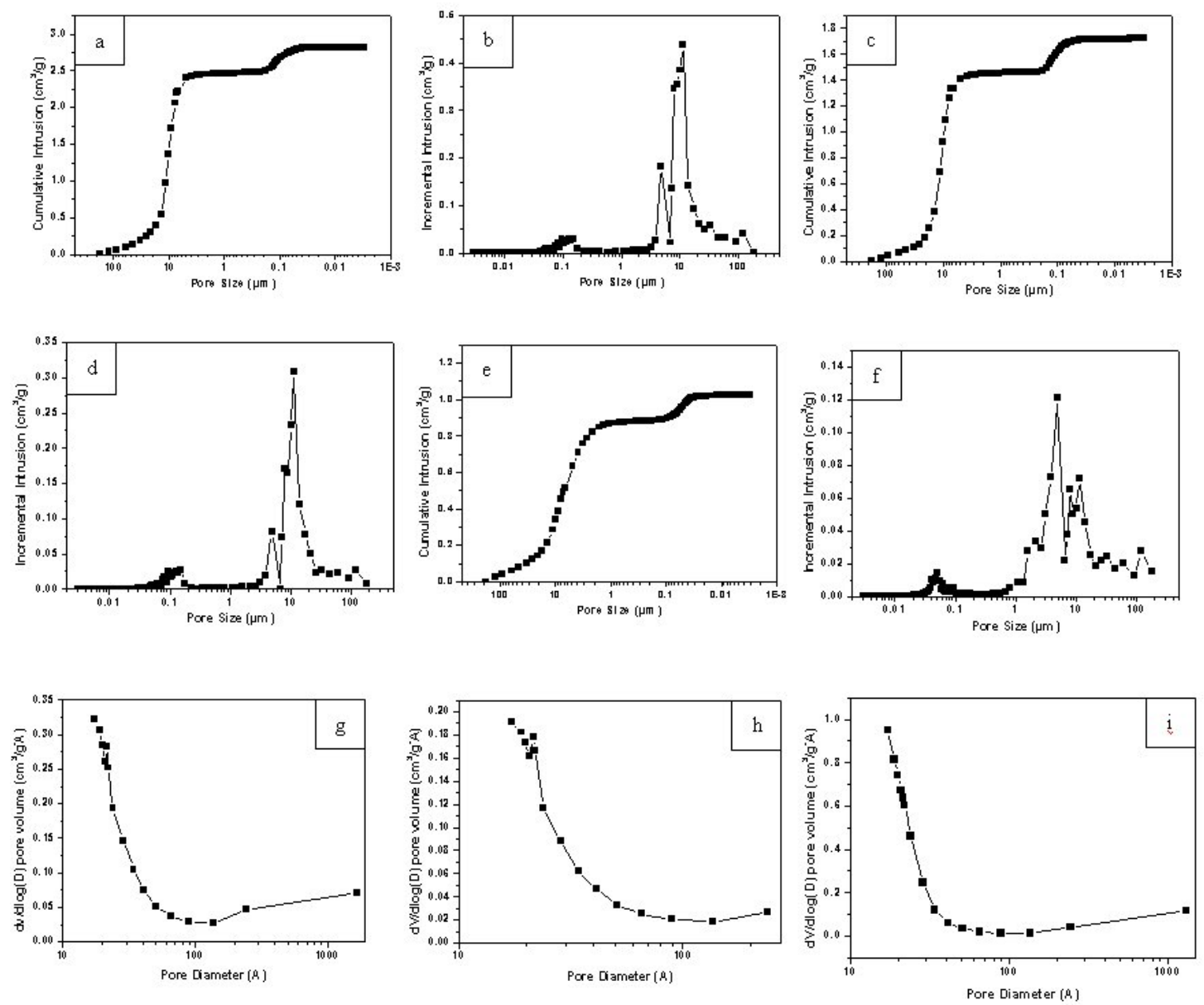

Figure S4: Silica Beads (a) Sample SiO-1, mercury cumulative intrusion plot; (b) Sample SiO-1, mercury incremental intrusion plot; (c) Sample SiO-2, mercury cumulative intrusion plot; (d) Sample SiO-2, mercury incremental intrusion plot; (e) Sample SiO-3, mercury cumulative intrusion plot; (f) Sample SiO-3, mercury incremental intrusion plot; (g) sample SiO-1: BJH pore size distribution plot; (h) Sample SiO-2: BJH pore size distribution plot; (i) Sample SiO-3: BJH pore size distribution plot. 

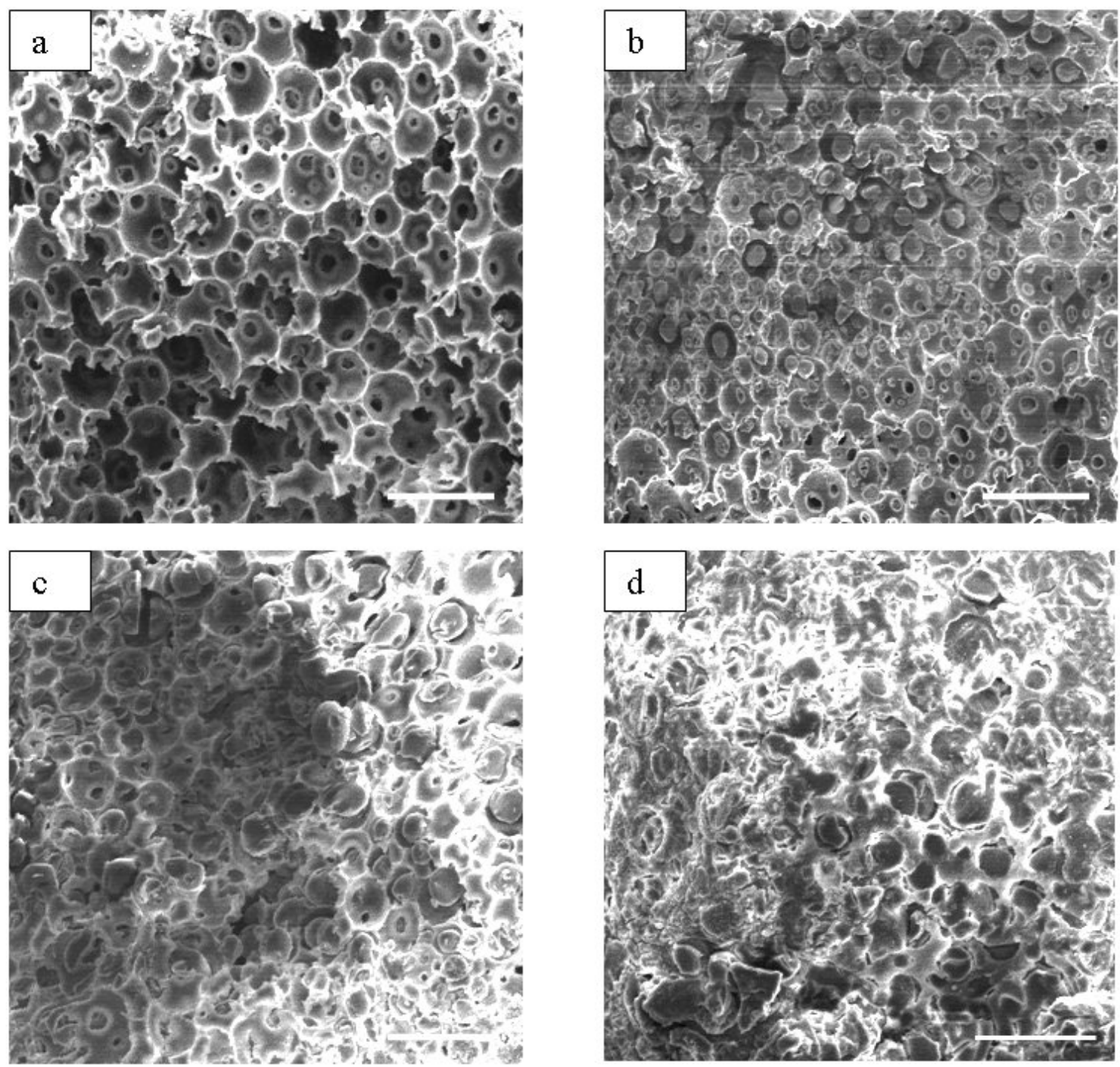

Figure S5: SEM images of alumina-polymer composite beads (a-b) and calcined alumina beads (c-d): (a) Internal pore structure of composite beads which were immersed in $\mathrm{Al}(\mathrm{O}-\mathrm{s}-\mathrm{Bu})_{3}$ solution once; mass gain $=42.50 \%$ (see Table 1 ), scale bar $=$ $100 \mu \mathrm{m}$; (b) Internal structure of composite beads C-AlO-1 which were obtained by immersing the above composite beads once again in precursor solution. Alumina microparticles can be observed entrapped in the emulsion-templated cells. Scale bar = $100 \mu \mathrm{m}$; (c) Internal structure of alumina beads AlO-1 which were obtained by calcining composite beads C-AlO-1. Very similar structures are observed before and after calcination. Scale bar $=100 \mu \mathrm{m}$; (d) Surface structure of alumina beads AlO-2. Scale bar $=100 \mu \mathrm{m}$. 

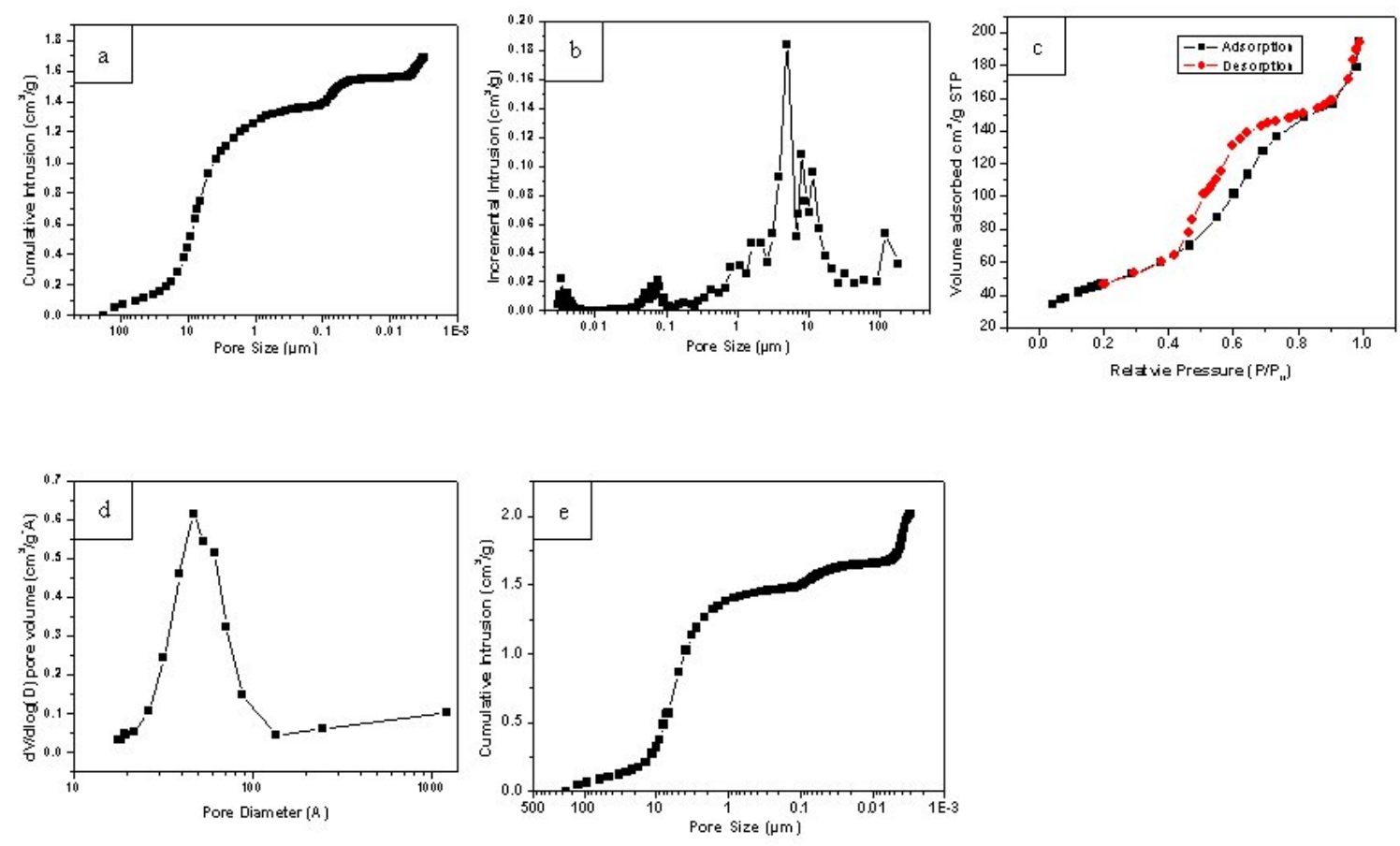

Figure S6: Alumina beads (a) Sample AlO-1, mercury cumulative intrusion plot; (b) Sample AlO-1, mercury incremental intrusion plot; (c) Sample AlO-1, BET isothermal plot; (d) Sample AlO-1, BJH pore size distribution plot; (e) Sample AlO-2,_mercury cumulative intrusion plot. 

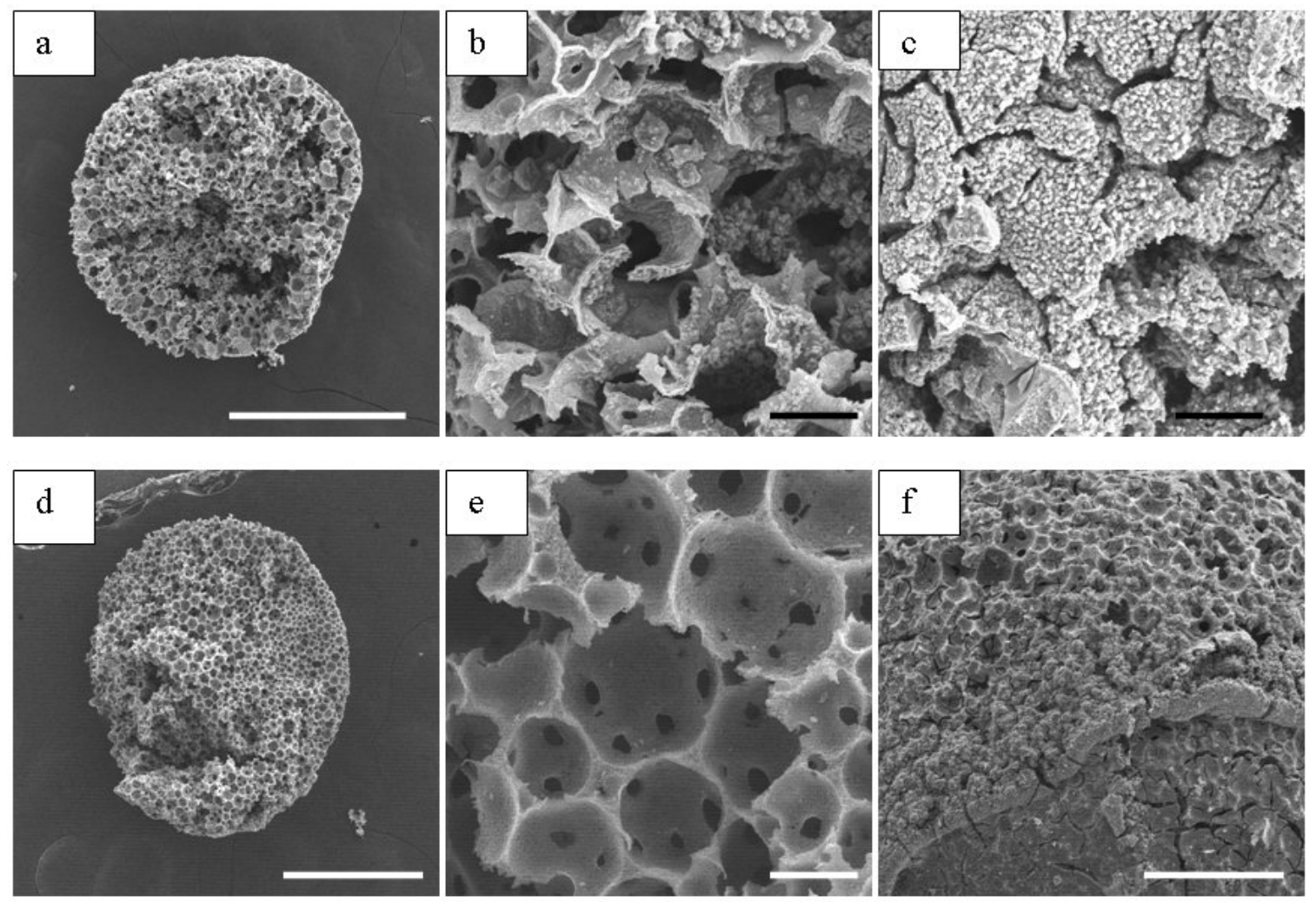

Figure S7: SEM images of titania beads $(\mathbf{a}-\mathbf{c})$ : Titania beads calcined from composite beads which were immersed directly into $\mathrm{Ti}(\mathrm{OiPr})_{4}$; (a) Cross-sectioned surface of one bead, showing porous structure, scale bar $=500 \mu \mathrm{m}$; (b) Internal structure at a higher magnification, scale bar $=25 \mu \mathrm{m}$; (c) Surface structure composed of dense titania particles, blocking the surface macropores, scale bar $=25 \mu \mathrm{m}$; $(\mathbf{d}-\mathbf{f})$ : Titania beads TiO-1: (d) Cross-sectioned surface of one bead, scale bar $=500 \mu \mathrm{m}$; (e) Internal pore structure shown at a higher magnification. The pores are interconnected. Scale bar $=25 \mu \mathrm{m}$; (f) Surface structure of a bead. Some macropores are blocked with titania. Scale bar = $200 \mu \mathrm{m}$. 

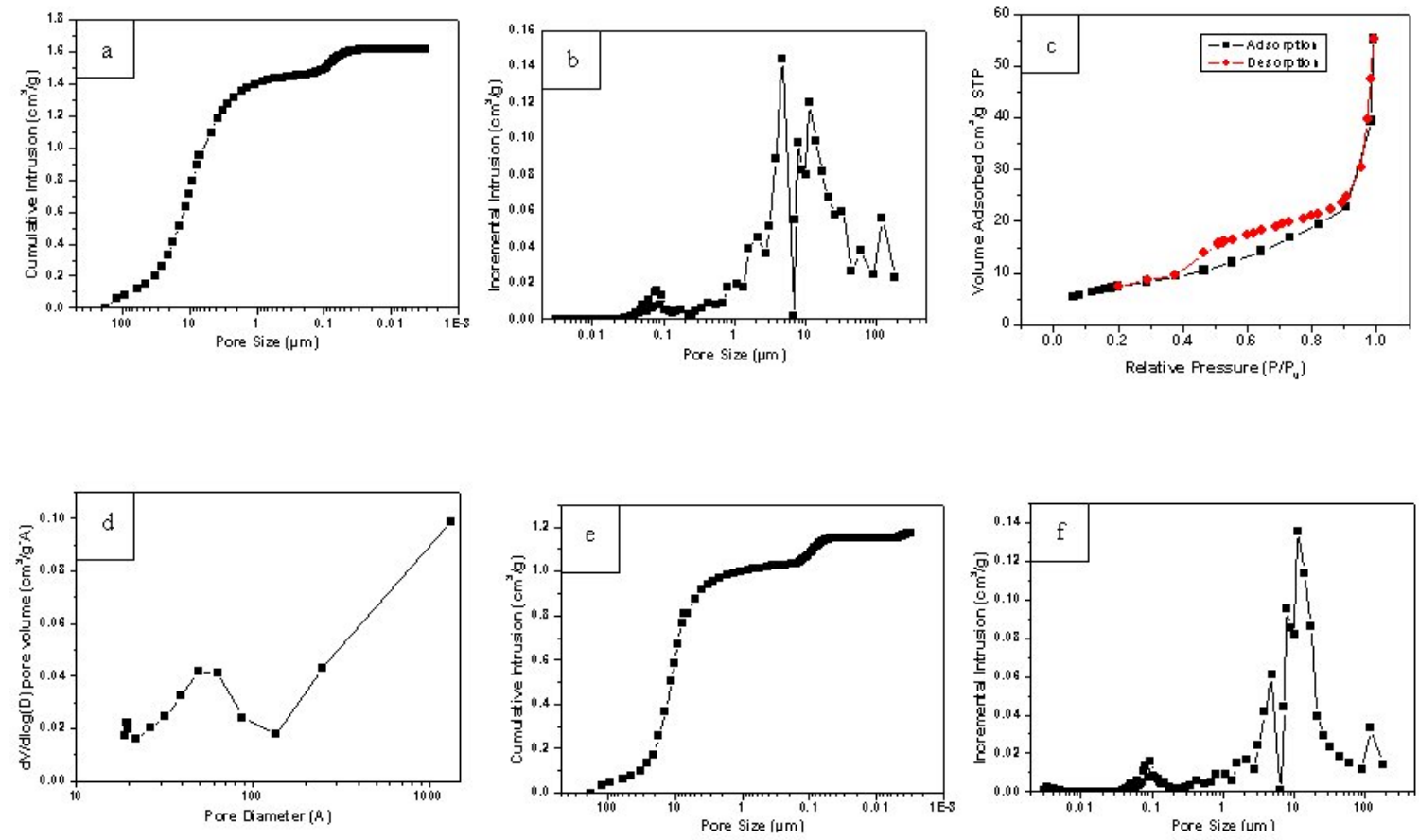

Figure S8: Titania beads (a) Sample TiO-1, mercury cumulative intrusion plot; (b) Sample TiO-1, mercury incremental intrusion plot; (c) Sample TiO-1, BET isothermal plot; (d) Sample TiO-1, BJH pore size distribution plot; (e) Sample TiO-2, mercury cumulative intrusion plot; (f) Sample TiO-2, mercury incremental intrusion plot. 

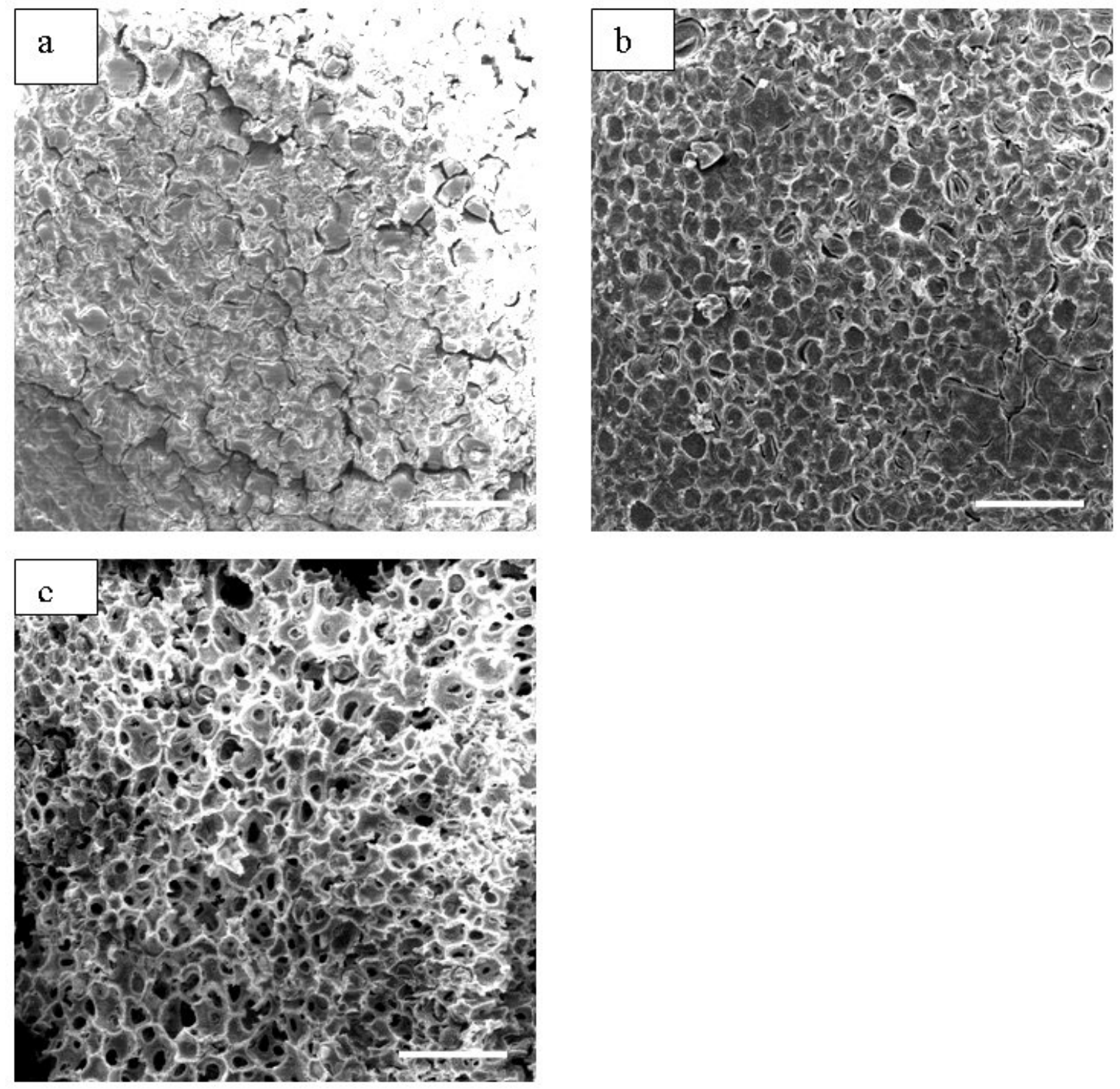

Figure S9: SEM images of zirconia-PAM composite beads (a) and zircona beads after calcinations (b,c) (a) Surface structure of composite beads C-ZrO-1, scale bar = $100 \mu \mathrm{m}$; (b) Surface structure of calcined zirconia beads ZrO-1, scale bar $=50 \mu \mathrm{m}$; (c) Internal pore structure of zirconia beads ZrO-1, scale bar $=100 \mu \mathrm{m}$. 

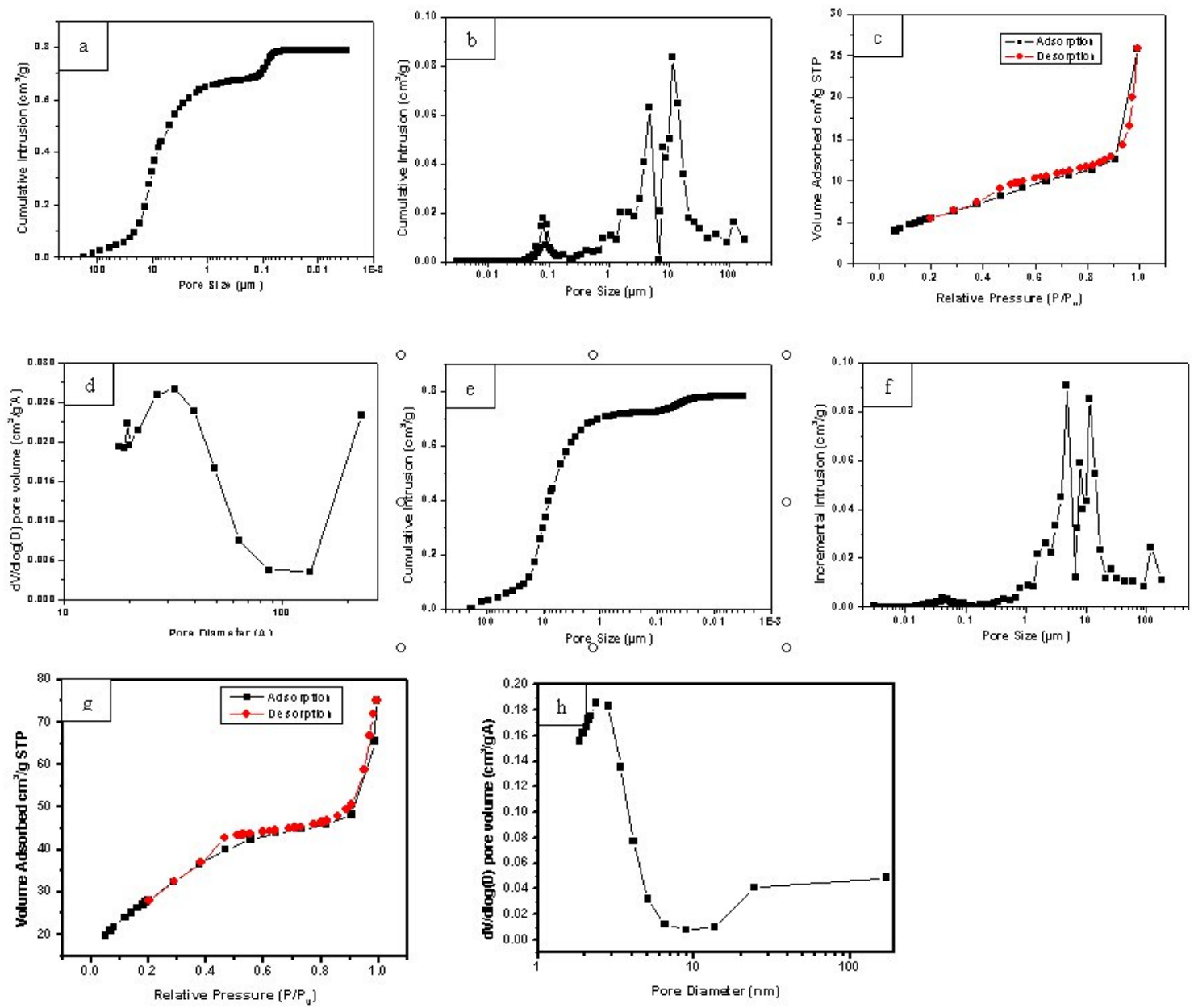

Figure S10: Zirconia beads (a) Sample ZrO-1, mercury cumulative intrusion plot; (b) Sample ZrO-1, mercury intrusion incremental plot; (c) Sample ZrO-1, BET isothermal plot; (d) Sample ZrO-1, BET pore size distribution plot; (e) Sample ZrO-2, mercury cumulative intrusion plot; (f) Sample ZrO-2, mercury incremental intrusion plot; (g) Sample ZrO-2, BET isothermal plot; (h) Sample ZrO-2, BJH pore size distribution plot. 